\title{
A first-principles study of pristine and Al-doped activated carbon interacting with 5-Fluorouracil anticancer drug
}

\author{
G. Román ${ }^{1}$, E. Noseda Grau ${ }^{1,2}$, A. Díaz Compañy ${ }^{1,2}$, G. Brizuela ${ }^{1}$, A. Juan ${ }^{1}$, and S. Simonetti ${ }^{1,3, a}$ \\ 1 Instituto de Física del Sur (IFISUR), Departamento de Física, Universidad Nacional del Sur (UNS), CONICET, \\ Av. L. N. Alem 1253, B8000CPB, Bahía Blanca, Argentina \\ 2 Comisión de Investigaciones Científicas (CIC), Calle 526 e/10 y 11, 1900, La Plata, Argentina \\ 3 Universidad Tecnológica Nacional (UTN), 11 de Abril 461, B8000LMI, Bahía Blanca, Argentina
}

Received 11 June 2018 and Received in final form 7 August 2018

Published online: 19 September 2018

(C) EDP Sciences / Società Italiana di Fisica / Springer-Verlag GmbH Germany, part of Springer Nature, 2018

\begin{abstract}
The adsorption of the 5-Fluorouracil (5-FU) molecule on the pristine and Al-doped activated carbon (AC) was investigated by using the Vienna Ab-initio Simulation Package. It is found that the 5-FU molecule is only weakly adsorbed on the pristine $\mathrm{AC}$ with high adsorption energy and large surface distance. The adsorption of the 5-FU molecule on pristine AC is highly disfavored. In contrast, the molecule shows strong interactions with the Al-doped AC confirmed by the lesser adsorption energy, the charge transfers on the Al-modified zone and the significant changes in the DOS at the Fermi level. The results of our study suggest that the $\mathrm{Al}$ dopant increases the adsorption capacity of $\mathrm{AC}$ enhancing its interactions with polar atoms of the adsorbate, hence improving its adsorption properties.
\end{abstract}

\section{Introduction}

5-Fluorouracil (5-FU) is one of the most successful agents in the medication of stomach, colorectal carcinoma, neck and head cancers $[1,2]$. Regardless of the improvement made with the introduction of new cytotoxic agents and medical training, the adsorption properties of this drug on the selected carrier matrix need to be investigated for more efficient therapies. Treatment with classical drugs such as 5-FU, has important harmful side effects. Thus, encapsulation in delivery systems may help to decrease side effects and admit oral administration. Oral drug administration presents numerous advantages, including patient's tolerance and the lower costs related to drug preparation and dispensation [3]. The efficacy of 5 -FU therapy may also be increased and its toxicity diminished by linking it with delivery systems that selectively transmit this active agent and reduce its toxicity [4]. In addition, the carrier may allow drugs to be liberated in a controlled way to the cancer zone preventing drug degradation [3-5].

Activated carbon (AC) presents significant pore area and dissemination, being an extraordinary material for adsorption processes [6]. In consequence, it is important to understand the advantages and inconvenience of 5-FU adsorption on AC to drug delivery applications. The effects of 5-FU adsorbed by activated carbon nanoparticles

\footnotetext{
a e-mail: ssimonet@uns.edu.ar
}

(ACNP) in vivo and in vitro, and the mechanism of ACNP to influence the anti-tumor effects of 5-FU has been studied [7]. ACNP can absorb 5-FU effectively and the absorption curve is consistent with exponential form. In vitro ACNP can block the cell cycle in the $\mathrm{S}$ phase, induce the prophase apoptosis and enhance the inhibitory effects of 5-FU on BGC-823 cells showing good concentration- and time-effect relationship. ACNP can block the cell cycle in the $\mathrm{S}$ phase, and absorbing 5 -FU can induce the prophase apoptosis. In vivo ACNP can inhibit the growth of tumor in $\mathrm{S}$ and prolong the life span of $\mathrm{H}$ bearing mice. ACNP can enhance the anti-cancer effect of 5 -FU in vivo and in vitro, induced the prophase apoptosis; and blocking the cell cycle in the $\mathrm{S}$ phase may be parts of the mechanisms of such effect.

Determining the structural properties of 5 -FU on the carbon surface leads to a better understanding of its bonding and reactivity during adsorption. On the other hand, the presence of a heteroatom on the carrier surface could have a significant effect on the adsorption. Doped materials are investigated in many applications such as biosensing, biological separation and anticancer therapy because of their novel properties as well as their extreme different functionality compared to their bulk counterparts. These unique properties distinguish them as potentially attractive candidates for a wide range of biological applications and innovative tools in the nanomedicine domain. Unfortunately their biomedical applications are almost unex- 
plored from the theoretical point of view. During the past few years many attempts have been made to investigate the interaction of carbon surfaces with different molecules. It is noteworthy that research on functionalized carbon surfaces is nowadays a hot spot for scientists. Hazrati and Hadipour have examined the ability of C60 fullerene to adsorb 5-FU [8]. The interactions of this drug with doped fullerenes showed that Al-doped C60 fullerene presents the most stable configuration [8]. On the other hand, Al-doped $\mathrm{B}_{24} \mathrm{~N}_{24}$ nanoclusters are also presented as a good strategy to increase the 5 -FU delivery performances because of a significant increase in the interaction strength and the electronic sensitivity $[9,10]$. In this work, the interaction of the 5-FU drug with an Al-doped activated carbon surface is studied by the density functional theory (DFT) in order to shed more light on this promising delivery system. The density functional theory (DFT) is widely applied in molecular modeling studies to predict and display structures and energy changes of small, medium-sized, and large molecules in chemistry. The methodology and model used in this paper are described in the next section.

\section{Computational methodology and model}

As the basic quantum-chemical approach, we selected the density functional theory (DFT). This is one of the most efficient tools of quantum chemistry that enables one to calculate structural and energetic properties of relatively large systems. In this work, DFT calculations were performed using the Vienna Ab-initio Simulation Package (VASP) $[11,12]$ including the dispersion interaction via Grimme's -D2 correction [13]. In this code plane-wave basis sets are used to solve the Kohn-Sham equations. The electron projector augmented-wave (PAW) method was used and the generalized gradient approximation (GGA) with the Perdew-Burke-Ernzerhof (PBE) functional was utilized [14-19]. The fixed convergence of the plane-wave expansion was found with cut-off energy of $400 \mathrm{eV}$. A set of $3 \times 3 \times 1$ Monkhorst-Pack k-points was used to sample the Brillouin Zone [20]. The ground state was found by a Methfessel-Paxton smearing of $0.2 \mathrm{eV}$ [21].

Some theoretical studies of graphite [22-27] have confirmed that most of its physics is the same as that of a single graphite layer. In theoretical studies, the structural models that are traditionally used to simulate adsorption are derived from graphite, in which all the atoms are in hexagonal rings [28]. The carbon pores are then assumed to have a slitlike shape confined by the parallel planes of the graphite. Accordingly, a slab consisting of three graphene layers arranged in a regular hexagonal pattern was modeled. The number of layers in the slab was previously tested. The criteria were followed to get faster convergence and a reasonable compromise between accuracy and computational time. In graphitic crystalline regions, the layers are stacked parallel to one another in a regular fashion. The atoms within a plane are covalently bonded (C-C bond length of $1.42 \AA$ ) with $s p^{2}$ hybridization, while the interaction between the sheets occurs through relatively weak van der Waals forces, giving
Table 1. Minimum distances and energies when 5-FU adsorbs on Al-doped activated carbon.

\begin{tabular}{|c|c|c|c|}
\hline $\begin{array}{c}\text { Geometry } \\
\text { (fig. 1) }\end{array}$ & Bond & Distance $(\AA)$ & $\Delta E(\mathrm{eV})$ \\
\hline A & Al-N & 2.09 & -2.30 \\
\hline B & Al-O & 1.83 & -2.74 \\
\hline C & Al-H & 2.52 & -1.95 \\
\hline D & Al-F & 1.99 & -2.23 \\
\hline
\end{tabular}

rise to a space between two graphene layers in graphite of about $3.35 \AA$ [29]. In a second stage, the slab was doped with $\mathrm{Al}$ atom by $\mathrm{C}$ atom substitution in graphite ring, obtaining an $\mathrm{Al}$ atoms concentration by surface area of $1 \mathrm{Al}$ atom $/ 144 \AA^{2}$. During relaxation, the bottom layer of the slab are kept fixed in bulk positions to represent the semi-infinite bulk beneath the surface while the others was fully relaxed together with the drug molecule. The adsorption energy $\left(E_{\text {ads }}\right)$ was calculated as the difference between the energy of the adsorbed molecule and the sum of the free surface and the isolated molecule energies. A negative adsorption energy value indicates an exothermic sorption process. When analyzing the possible structures of the molecule adsorption complexes, we have selected diverse arrangements of the adsorbate molecule on the surface to ensure that we identified the lowest-energy adsorbate/substrate structures. Here we present and discuss the most stable geometries obtained.

\section{Results and discussion}

According to the results of our calculations, only a weak interaction occurs between 5-FU and the pristine $\mathrm{AC}$, which resulted in adsorption energy of $-0.20 \mathrm{eV}$. Indeed, the high $E_{\text {ads }}$ value and the long molecule-surface distances $(d=3.10 \AA)$ suggest that 5 -FU molecules are not significantly adsorbed on pristine AC surface, and only undergo a physical adsorption by weak van der Waals interactions between the molecule and the surface. Therefore, pristine $\mathrm{AC}$ could not be able to bind 5-FU strongly enough to adsorb it from drug solution.

However, the interaction of the 5-FU ring with the Aldoped AC surface leads to more negative-energy values (see table 1), suggesting that the interaction is enhanced in comparison with the pure surface. 5 -FU is adsorbed on the $\mathrm{Al}$ zones that are favorable enough to interact with the polar atoms of the 5-FU molecule. Figure 1 shows the obtained most stable geometries. Since the $\mathrm{Al}$ atom is bigger than the $\mathrm{C}$ atom, after full optimization it is found that the $\mathrm{Al}$ atom protrudes out of the plane and causes a drastic distortion in the region around the doping site to relieve the stress. This causes that the bond lengths at the doping site are elongated to $d_{\mathrm{Al}-\mathrm{C}}=1.85-1.87 \AA$ from $d_{\mathrm{C}-\mathrm{C}}=1.42 \AA$. This variation in bond lengths is related to the distortion of hexagonal structures adjacent to the $\mathrm{Al}$ atom, which was also observed in Al-doped carbon nanostructures [30-32]. For the Al-doped AC system, 


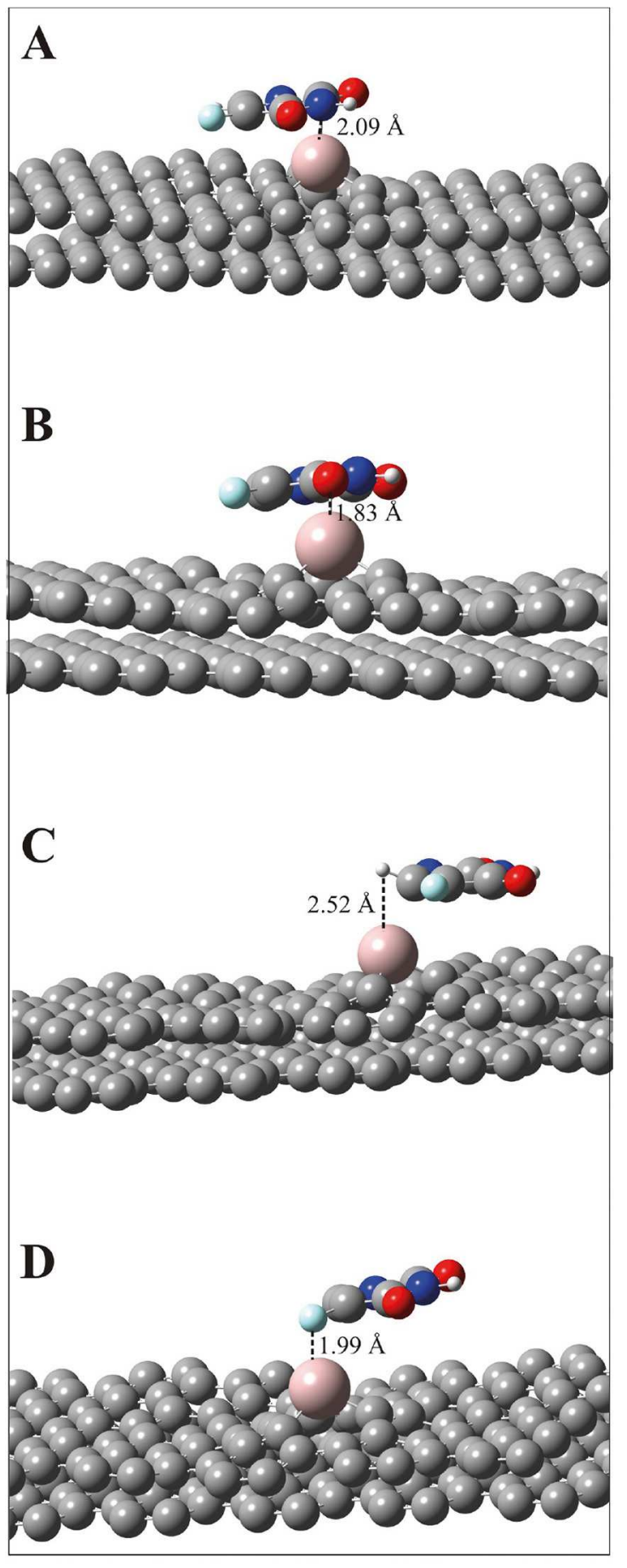

Fig. 1. Major geometries for 5-FU adsorbed on Al-doped activated carbon (for simplicity only two graphene layers are shown).

the most favored state is the doublet, which indicates that the missing electron of the isolated $\mathrm{Al}$ atom couples with one of the two electrons of the graphene sheet (with the $\mathrm{C}$ monovacance), leaving the other electron dispersed on the substrate. A relevant electronic transfer was observed between $\mathrm{Al}$ and the three neighboring $\mathrm{Cs}$ of about $0.8 e$ with respect to pristine AC [33]. Regarding the location

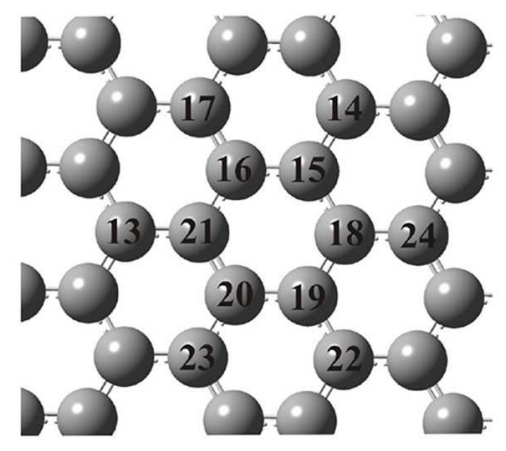

AC surface

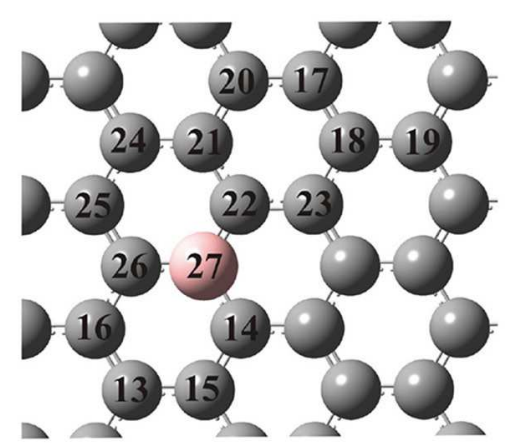

Al-doped AC surface

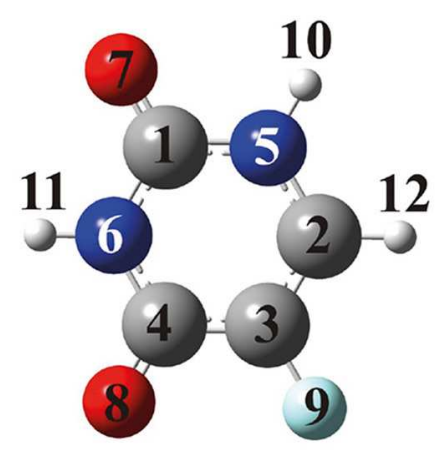

$5-\mathrm{FU}$

Fig. 2. Reference for atoms (table 2 and table 3 ).

of the spin, approximately 0.4 of the total spin density corresponding to the doublet configuration is located on the $\mathrm{Al}$ and its first neighbors. Comparing this result with the fraction obtained for the regular surface $(0.25)$, it can be observed that the spin is more localized in the case of the adsorption in the $\mathrm{Al}$ region; this positively influences the absorption of the 5-FU molecule. Among the positions of minimum energy adopted for 5 -FU on the doped surface, when the molecule absorbs through the $\mathrm{Al}-\mathrm{O}$ interaction, it was found that this geometry leads to the major ring adsorption (see table 1). A similar interaction has been obtained for 5-FU adsorption on doped C60 fullerene for an Al-O distance of $1.8 \AA$ [8]. In addition, the O-side of the 5-FU molecule implies strong adsorption 
Table 2. Partial charges on atoms when 5-FU adsorbs on pristine activated carbon (AC).

\begin{tabular}{|c|c|c|c|}
\hline $\begin{array}{c}\text { Atom } \\
\text { (ref. fig. 2) }\end{array}$ & Isolated 5-FU & Isolated AC & $5-\mathrm{FU}$ on $\mathrm{AC}$ \\
\hline 1 & 0.000 & & 0.000 \\
\hline 2 & 2.914 & & 2.911 \\
\hline 3 & 3.165 & & 3.334 \\
\hline 4 & 1.357 & & 1.231 \\
\hline 5 & 7.984 & & 8.000 \\
\hline 6 & 8.025 & & 7.964 \\
\hline 7 & 7.937 & & 7.968 \\
\hline 8 & 7.811 & & 7.885 \\
\hline 9 & 7.861 & & 7.815 \\
\hline 10 & 0.000 & & 0.000 \\
\hline 11 & 0.000 & & 0.000 \\
\hline 12 & 0.942 & & 0.914 \\
\hline 13 & & 3.931 & 4.030 \\
\hline 14 & & 4.015 & 4.100 \\
\hline 15 & & 4.022 & 4.027 \\
\hline 16 & & 3.922 & 3.924 \\
\hline 17 & & 4.115 & 4.078 \\
\hline 18 & & 4.015 & 3.934 \\
\hline 19 & & 4.022 & 4.086 \\
\hline 20 & & 3.922 & 3.928 \\
\hline 21 & & 4.115 & 4.015 \\
\hline 22 & & 4.015 & 4.011 \\
\hline 23 & & 4.115 & 4.072 \\
\hline 24 & & 3.919 & 3.948 \\
\hline
\end{tabular}

on the metal (Al-, Ga- and Ge-) doped BNNTs [9]. Recent results also showed that mainly the $\mathrm{Al}$ atom in doped $\mathrm{B}_{24} \mathrm{~N}_{24}$ fullerene tends to have an interaction with the oxygen atoms of 5 -FU at $\mathrm{Al}-\mathrm{O}$ distances of $1.86 \AA$ and $1.89 \AA$, respectively [10].

The partial charge on atoms has been made to explore the changes in the electronic structure of the drug molecule after its attachment to the surface. Partial charges on the atoms when 5 -FU adsorbs on pristine $\mathrm{AC}$ and Al-doped AC, are shown in table 2 and table 3, respectively (see reference for atoms in fig. 2). When adsorption is produced on pristine activated carbon, small changes can be seen on 5 -FU and $\mathrm{C}$ surface atoms confirming the weak interactions between the ring molecule and carbon surface. When adsorption is produced on doped $\mathrm{AC}$, the major changes are shown on $\mathrm{C}$ atoms of the ring bonded to $\mathrm{Al}$ atom. Initially, a partial charge is transferred from the $\mathrm{Al}$ atom to the vicinal carbon atoms. Thus, the dopant site (Al atom) acts as an affinity center for the chemisorption of the 5-FU molecule. In consequence, the polar atoms of 5 -FU bind to the exposed $\mathrm{Al}$ atom which is electron-donant thus making the molecule more stabilized. Therefore, the $\mathrm{Al}$ atom at the $\mathrm{AC}$ surface is more reactive toward the electrophile attack of the 5 -FU
Table 3. Partial charges on atoms when 5 -FU chemisorbs on Al-doped activated carbon (Al-AC).

\begin{tabular}{|c|c|c|c|}
\hline $\begin{array}{c}\text { Atom } \\
\text { (ref. fig. 2) }\end{array}$ & Isolated 5-FU & Isolated Al-AC & 5 -FU on $\mathrm{Al}-\mathrm{AC}$ \\
\hline 1 & 0.000 & & 0.000 \\
\hline 2 & 2.914 & & 3.000 \\
\hline 3 & 3.165 & & 3.205 \\
\hline 4 & 1.357 & & 1.486 \\
\hline 5 & 7.984 & & 7.962 \\
\hline 6 & 8.025 & & 8.007 \\
\hline 7 & 7.937 & & 7.962 \\
\hline 8 & 7.811 & & 8.057 \\
\hline 9 & 7.861 & & 7.820 \\
\hline 10 & 0.000 & & 0.000 \\
\hline 11 & 0.000 & & 0.000 \\
\hline 12 & 0.942 & & 0.864 \\
\hline 13 & & 3.990 & 3.980 \\
\hline 14 & & 4.935 & 4.728 \\
\hline 15 & & 3.990 & 3.999 \\
\hline 16 & & 4.005 & 4.093 \\
\hline 17 & & 3.938 & 3.964 \\
\hline 18 & & 4.087 & 4.100 \\
\hline 19 & & 4.069 & 3.940 \\
\hline 20 & & 4.091 & 4.101 \\
\hline 21 & & 3.978 & 3.978 \\
\hline 22 & & 4.833 & 4.698 \\
\hline 23 & & 4.058 & 4.019 \\
\hline 24 & & 3.957 & 4.001 \\
\hline 25 & & 4.030 & 4.068 \\
\hline 26 & & 4.987 & 4.702 \\
\hline 27 & & 0.020 & 0.000 \\
\hline
\end{tabular}

molecule. In the doped zone, $\mathrm{Al}$ causes a rearrangement in the electron density and an increase in the strength of the interactions resulting in strong binding energies. Binding energies are found to be more negative implying stability towards covalent attachment of the drug molecule onto doped AC.

We have computed the density of states (DOS) of the system when 5-FU absorbs on the Al-doped AC surface. For comparison, the densities of states of the clean Al-doped AC surface, the isolated 5-FU molecule and the pristine AC surface were also calculated (see fig. 3). There are bands associated with the interaction between 5-FU and the Al-doped surface orbitals. The overlapping molecule-surface takes mainly place within -6 and $-18 \mathrm{eV}$ and near the Fermi level. 5-FU mainly contributes with new states in the low part of the band in the range of -24 to $-31 \mathrm{eV}$. Replacing a carbon atom by an aluminum atom causes changes in the DOS spectrum compared with the pristine AC. New states appear between $-1 \mathrm{eV}$ and the Fermi level which belong to the $2 s$ and $2 p$ orbitals of the $\mathrm{Al}$ atom (see fig. 3 (right)). A more uniform populated region 


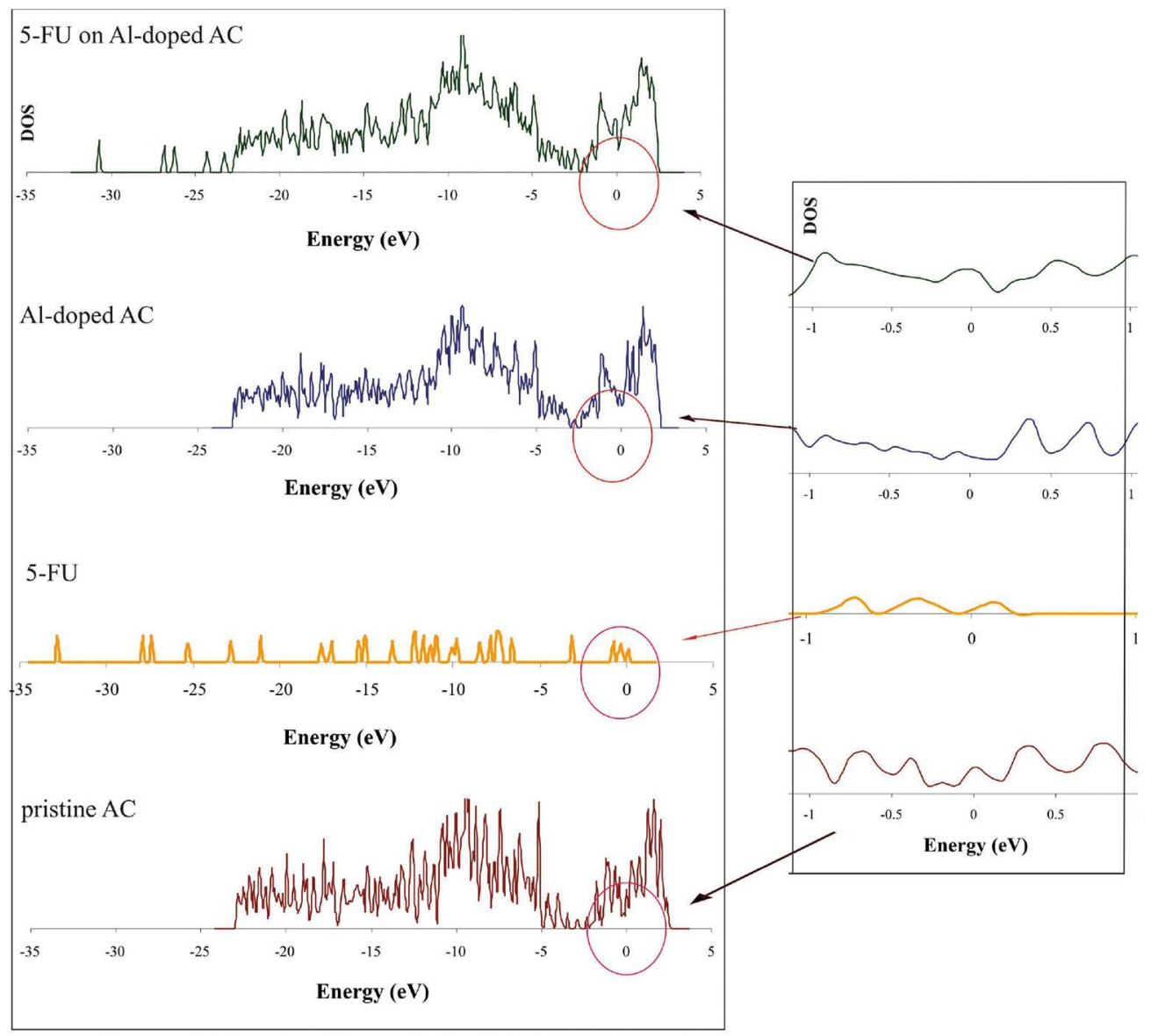

Fig. 3. Density of states (DOS) of 5-FU adsorbed on Al-doped AC surface, Al-doped AC surface, 5-FU molecule and pristine AC surface (left from top to bottom). Amplified DOS at Fermi level is shown (right).

can now be seen near the Fermi level. Consequently, electrons are transferred more easily from the valance level to the conducting level during drug adsorption. The $\mathrm{Al}$ atom gives rise to a large increase in the electrical conductivity of the graphene layer. The electronic charge strongly overlaps which leads to more orbital mixing and a large charge transfer. These observations clarify the responsibility of the $\mathrm{Al}$ atom in the charge transfer process during the adsorption of drug and support the strongest interaction of the 5-FU molecule with Al-doped AC.

\section{Conclusions}

Our computational study contributes to open new routes to design efficient materials as drug delivery systems. The 5 -FU molecule does not adsorbed efficiently on the pristine AC surface. This claim is confirmed by the high adsorption energy and the big molecule-surface distance. It is found that the 5-FU molecule major stabilized on $\mathrm{Al}-$ doped surface. The molecule remarkably tends to adsorb via its polar atoms with an appreciable strong adsorption energy. The smaller adsorption energy, the charge transfers on the Al-doped zone and the significant changes in the DOS at the Fermi level confirm the results and open a new perspective for designing novel drug delivery systems based on Al-doped carbon surfaces.

Our work was supported by SCyT UTN, SCyT UNS, CIC Bs. As. and CONICET. AJ, GB and SS are members of CONICET. ADC is member of CIC Bs. As. GR is a fellow of CONICET. ENG is a fellow of CIC Bs. As.

\section{Author contribution statement}

All authors contributed equally to this work.

\section{References}

1. T.A. Rich, R.C. Shepard, S.T. Mosley, J. Clin. Oncol. 22, $2214(2004)$.

2. X. Wang, J. Lin, X. Zhang, Q. Liu, Q. Xu, R.-X. Tan, Z. Guo, J. Inorg. Biochem. 94, 186 (2003).

3. F.H. Lin, Y.H. Lee, C.H. Jian, J.-M. Wong, M.-J. Shieh, C.-Y. Wang, Biomaterials 23, 1981 (2002).

4. J.L. Arias, Molecules 13, 2340 (2008).

5. Y. Zhu, T. Ikoma, N. Hanagata, S. Kaskel, Small 6, 471 (2010). 
6. R.C. Bansal, J.B. Donnet, F. Stoeckli, Active Carbon (Dekker, New York, 1988).

7. Y. Zhong, Z. Junxian, L. Peifeng, Z. Yingge, J. Sci. Conf. Proc. 1, 190 (2009) https://doi.org/10.1166/jcp.2009.1056.

8. M.K. Hazrati, N.L. Hadipour, Phys. Lett. A 380, 937 (2016).

9. A. Soltani, M.T. Baei, E.T. Lemeski, S. Kaveh, H. Balakheyli, J. Phys. Chem. Solids 86, 57 (2015).

10. M.K. Hazrati, Z. Javanshir, Z. Bagheri, J. Mol. Graph. Model. 77, 17 (2017).

11. http://www.vasp.at/.

12. G. Kresse, J. Hafner, Phys. Rev. B 47, 558 (1993).

13. S. Grimme, J. Comput. Chem. 27, 1787 (2006).

14. G. Kresse, J. Hafner, Phys. Rev. B 48, 13115 (1993).

15. G. Kresse, J. Hafner, Phys. Rev. B 49, 14251 (1994).

16. J.P. Perdew, J.A. Chevary, S.H. Vosko, K.A. Jackson, M.R. Pederson, D.J. Singh, C. Fiolhais, Phys. Rev. B 46, 6671 (1992).

17. J.P. Perdew, J.A. Chevary, S.H. Vosko, K.A. Jackson, M.R. Pederson, D.J. Singh, C. Fiolhais, Phys. Rev. B 48, 4978 (1993).

18. P. Bloch, Phys. Rev. B 50, 17953 (1994).

19. G. Kresse, D. Joubert, Phys. Rev. B 59, 1758 (1999).
20. H.J. Monkhorst, J.D. Pack, Phys. Rev. B 13, 5188 (1976).

21. M. Methfessel, A.T. Paxton, Phys. Rev. B 40, 3616 (1989).

22. N. Chen, R.T. Yang, Carbon 36, 1061 (1998).

23. N. Chen, R.T. Yang, J. Chem. Phys. A 102, 6348 (1998).

24. D. Lamoen, B.N.J. Persson, J. Chem. Phys. 108, 3332 (1998).

25. Z.H. Zhu, G.Q. Lu, Langmuir 20, 10751 (2004).

26. C. Janiak, R.R. Hoffmann, P. Sjovall, B. Kasemo, Langmuir 9, 3427 (1993).

27. J.R. Pliego, S.M. Resende, E. Humeres, J. Chem. Phys. 314, 127 (2005).

28. K.T. Thomson, K.E. Gubbins, Langmuir 16, 5761 (2000).

29. A.P. Terzyk, S. Furmaniak, P.A. Gauden, P.J.F. Harris, J. Włoch, P. Kowalczyk, J. Phys.: Condens. Matter. 19, 406208 (2007).

30. J.M. Hernández, E.C. Anota, M.T. de la Cruz, M.G. Melchor, G.H. Cocoletzi, J. Mol. Model. 18, 3857 (2012).

31. Z.M. Ao, J. Yang, S. Li, Q. Jiang, Chem. Phys. Lett. 461, 276 (2008).

32. R. Wang, D. Zhang, W. Sun, Z. Han, C. Liu, J. Mol. Struct. (THEOCHEM) 806, 93 (2007).

33. N. Domancich, Doctoral Thesis (2013) http:// repositoriodigital.uns.edu.ar/handle/123456789/ 2455. 